\title{
Synthesis and Dielectric Studies of Monoclinic Nanosized Zirconia
}

\author{
I. Flavia Princess Nesamani, ${ }^{1}$ V. Lakshmi Prabha, ${ }^{2}$ Aswathy Paul, ${ }^{1}$ and D. Nirmal ${ }^{1}$ \\ ${ }^{1}$ Department of Electronics and Communication Engineering, Karunya University, Coimbatore 641114, India \\ ${ }^{2}$ Department of Electronics and Communication Engineering, Government College of Technology, Coimbatore 641013, India
}

Correspondence should be addressed to I. Flavia Princess Nesamani; flavia@karunya.edu

Received 11 October 2013; Revised 22 December 2013; Accepted 5 January 2014; Published 16 February 2014

Academic Editor: Nian X. Sun

Copyright (C) 2014 I. Flavia Princess Nesamani et al. This is an open access article distributed under the Creative Commons Attribution License, which permits unrestricted use, distribution, and reproduction in any medium, provided the original work is properly cited.

\begin{abstract}
Zirconium dioxide is a prospective high- $\kappa$ material that can replace silicon dioxide. Zirconium dioxide nanoparticle has been synthesized using sol-gel process at room temperature. The structural and morphological characterization of the nanoscaled zirconium dioxide is done using FTIR, SEM, X-ray diffraction, and TEM. The particle size of the synthesized $\mathrm{ZrO}_{2}$ is observed in the range of 50-80 nm with an average crystallite size of 2-10 $\mathrm{nm}$. The results are compared with commercial coarse zirconia which showed a particle size in the range of $900 \mathrm{~nm}-2.13 \mu \mathrm{m}$ and crystallite size of $5.3 \mathrm{~nm}-20 \mathrm{~nm}$. It is expected that both nanoscaling and the high dielectric constant of $\mathrm{ZrO}_{2}$ would be useful in replacing the low- $\kappa \mathrm{SiO}_{2}$ dielectric with high- $\kappa \mathrm{ZrO}_{2}$ for CMOS fabrication technology. The synthesized $\mathrm{ZrO}_{2}$ is subjected to impedance analysis and it exhibited a dielectric constant of 25 to find its application in short channel devices like multiple gate FinFETS and as a suitable alternative for the conventional gate oxide dielectric $\mathrm{SiO}_{2}$ with dielectric value of 3.9, which cannot survive the challenge of an end of oxide thickness $\leq 1 \mathrm{~nm}$.
\end{abstract}

\section{Introduction}

The ever growing semiconductor industry has turned towards nanoscaled devices with high- $\kappa$ metal oxide gate dielectrics along with tuneable metal gates in transistor manufacturing processes to meet the need for high speed transistors, while maintaining low power consumption. The best metal oxide to replace Silicon dioxide is hafnia or zirconia $\left(\mathrm{HfO}_{2} / \mathrm{ZrO}_{2}\right)$. The particles when synthesized in the magical dimension scale of nanometer [1] have wide applications with the improvement of the electrical, mechanical, and optical properties of any compound due to the increase in surface area. Zirconia, a metal oxide, is expected to be invariant with higher thermal expansion ratio in an FET configuration. A figure of merit to judge a high$\kappa$ gate dielectric layer is the equivalent oxide thickness $(\mathrm{EOT})=\left(\kappa_{\mathrm{SiO}_{2}} / \kappa_{\text {high- } \kappa}\right) \mathrm{d} \kappa$. If a dielectric material with a higher dielectric constant (high- $\kappa$ ) can replace $\mathrm{SiO}_{2}$, the dielectric layer thickness can be increased proportionally while keeping the same gate capacitance $[2,3]$. Since a thicker layer is used for insulation, the tunnelling current is drastically reduced in the tunnelling region. Meanwhile, before incorporating a new high $-\kappa$ material into the present ULSI process flow, many requirements, like particle size, capacitance at different thickness of the material, and so forth, need to be optimized [4-8]. One of the most crucial elements that allow the successful scaling is certainly the material and electrical properties of $\mathrm{ZrO}_{2}$ [4, 5, 9-11] offsets compared to the reported band gap of 5.16 to $7.8 \mathrm{eV}$ for $\mathrm{ZrO}_{2}$. Among the high $-\kappa$ materials, $\mathrm{ZrO}_{2}$ is a promising candidate in multiple gate FinFETS and a lot of research works focus on this material [2]. Pure zirconia exhibits three stages of polymorphism. The monoclinic, tetragonal, and cubical symmetries are stable up to the temperature range of $1100^{\circ} \mathrm{C}$, between 1100 and $2370^{\circ} \mathrm{C}$, and above $2370^{\circ} \mathrm{C}$, respectively. 


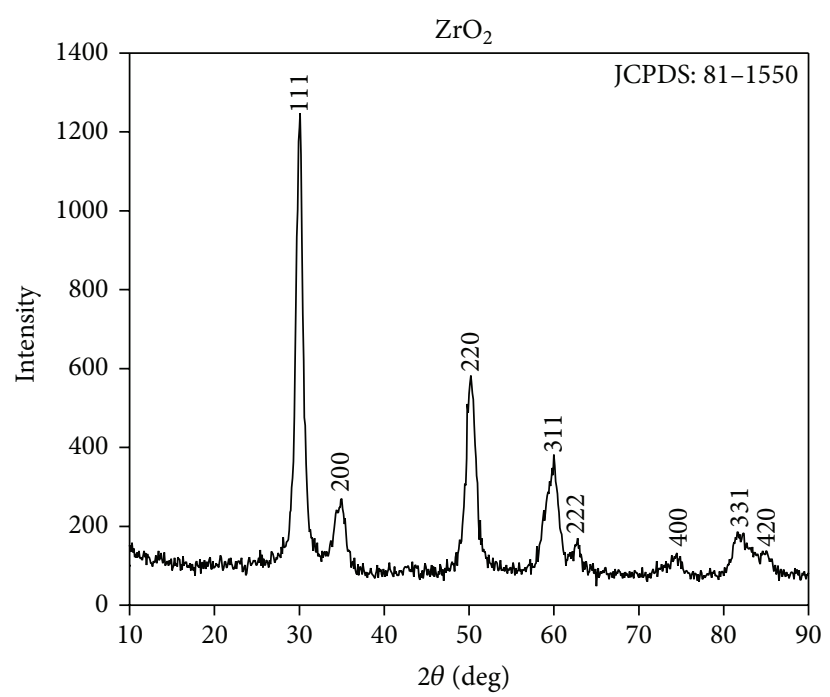

(a)

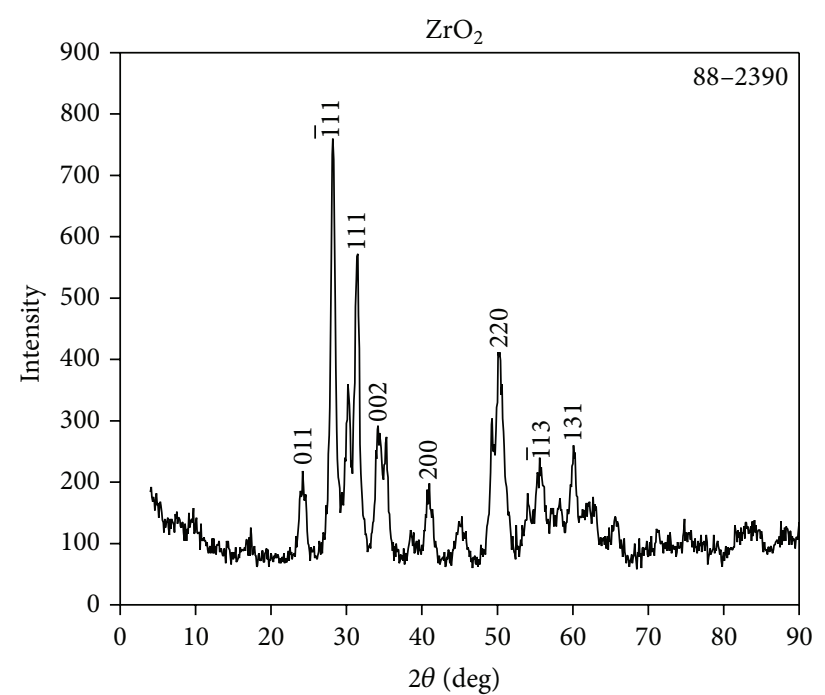

(b)

FIGURE 1: (a) and (b): XRD pattern of coarse zirconia and synthesized zirconia.

Zirconium dioxide, from the group of ceramic semiconductors, possesses special transition-metal oxide properties. The ceramic based zirconia is used in variety of applications such as technological and commercial applications, for example, catalyst, oxygen sensors, and high dielectric constant material for large scale integrated circuit. Zirconium dioxide has good thermal stability, high strength, low thermal conductivity, and high corrosion resistance. It is an excellent high- $\kappa$ gate oxide dielectric widely accepted in high- $\kappa /$ metal gate stacks for CMOS fabrication technology. Zirconium oxide can be prepared by sol-gel technique (Brinker and Scherer, 1990 [12]) using inorganic precursors like $\mathrm{ZroCl}_{2}$ or metal organic precursors like zirconium alkoxides [13]. Considering the development of new materials, the experimental conditions can be adjusted in order to get stable or metastable compounds. Most studies performed in the field focus on the synthesis of materials with mixed composition [14-17]. The physical and chemical properties of mixed oxides prepared through sol-gel process are of great interest due to their thermal and chemical stability. Besides they are highly acidic because of the presence of $\mathrm{OH}$ groups on the surface [18]. Stable dispersions, or sols, of small particles (less than 0.1 micrometre) are formed from precursor chemicals such as metal alkoxides or other metalorganics [18]. By partial evaporation of the liquid or addition of a suitable initiator, a polymer-like, threedimensional bonding takes place within the sol to form a gelatinous network or gel. The gel can then be dehydrated and calcined to obtain a fine, intimately mixed ceramic powder. This paper presents the synthesis using solgel process and material characterization of the nanoscaled zirconia through FTIR, SEM, TEM, and XRD analysis along with the evaluation of dielectric constant through impedance spectroscopy test.

\section{Experimental}

2.1. Synthesis Method. In this work, one of the widely used cost effective sol-gel method $[1,5,14,16,18]$ is employed for the preparation of $\mathrm{ZrO}_{2}$. The powders with different particle size, morphology, and phase composition can be prepared by varying parameters such as temperature, duration of the processing, and concentration of chemical species to solution [1]. $15 \mathrm{mmol}$ of zirconium oxychloride $\left(\mathrm{ZrOCl}_{2}\right.$ Sigma Aldrich) is dissolved in distilled water and aqueous ammonia is added drop by drop under constant stirring using magnetic stirrer to form gel-like precipitate until it reached a $\mathrm{pH}$ value of 10 . The chemical equation is given as

$$
\begin{gathered}
\mathrm{ZrOCl}_{2}+2 \mathrm{H}_{2} \mathrm{O}+2 \mathrm{NH}_{3} \frac{\text { Benzyl Alcohol Surfactant }}{\mathrm{pH} 10} \mathrm{ZrO}_{2}+2 \mathrm{NH}_{4} \mathrm{Cl}+\mathrm{H}_{2} \mathrm{O} \\
\mathrm{ZrO}_{2} \text { (microparticles) } \stackrel{\text { Sodium dodecyl Sulphate }}{\longrightarrow} \mathrm{ZrO}_{2} \text { (nanoparticles) }
\end{gathered}
$$

$30 \mathrm{mmol}$ of benzyl alcohol was added as surfactant to reduce the surface tension. $30 \mathrm{mmol}$ of sodium dodecyl sulphate is used as phase transition catalyst to form nanoparticle size. Then, the precipitate is filtered through high grade Whattman filter of nanopore size and thoroughly washed with water until elimination of chloride using deionized water. The precipitate is then dried in an oven for $24 \mathrm{hr}$ at $120^{\circ} \mathrm{C}$. The solid zirconium dioxide is calcined at $750^{\circ} \mathrm{C}$ for $2 \mathrm{hrs}$ twice to obtain zirconium dioxide in the white powder form. 


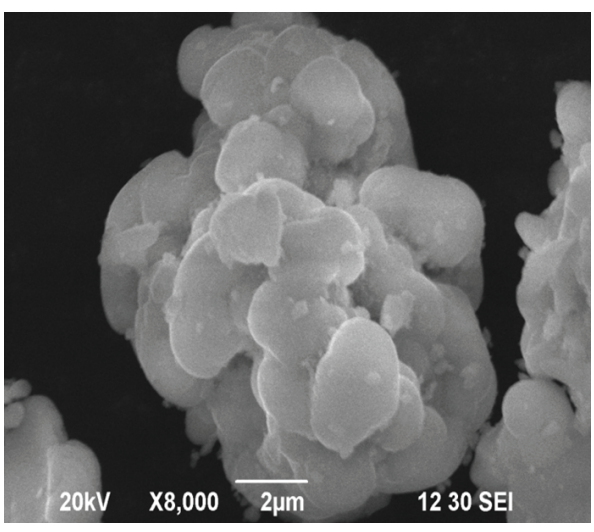

(a)

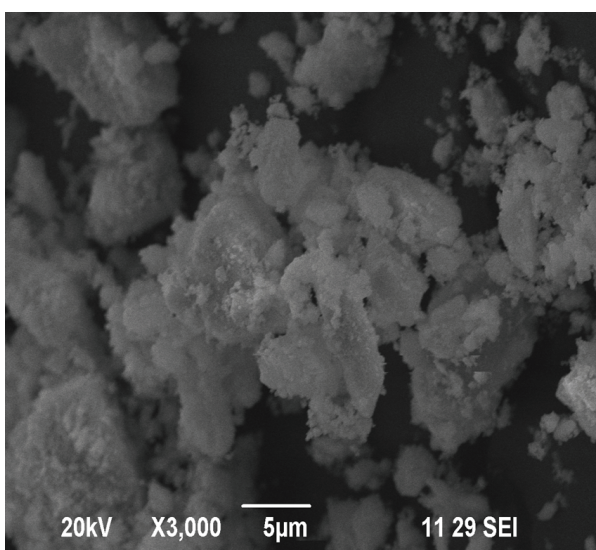

(c)

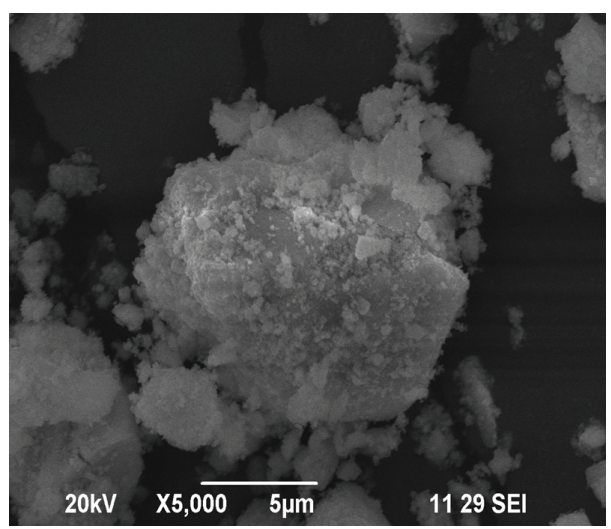

(e)

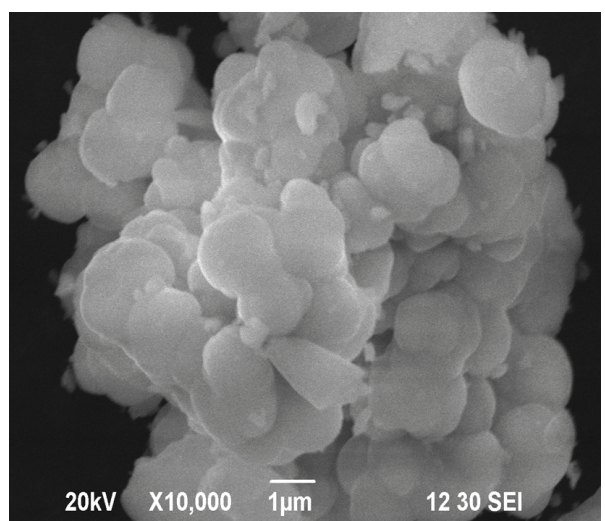

(b)

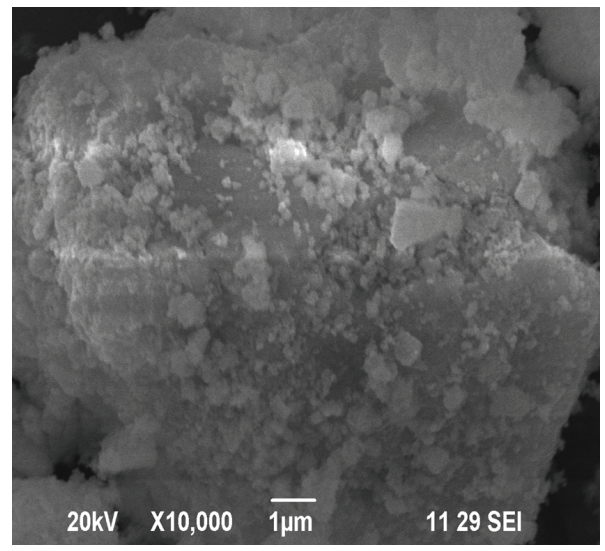

(d)

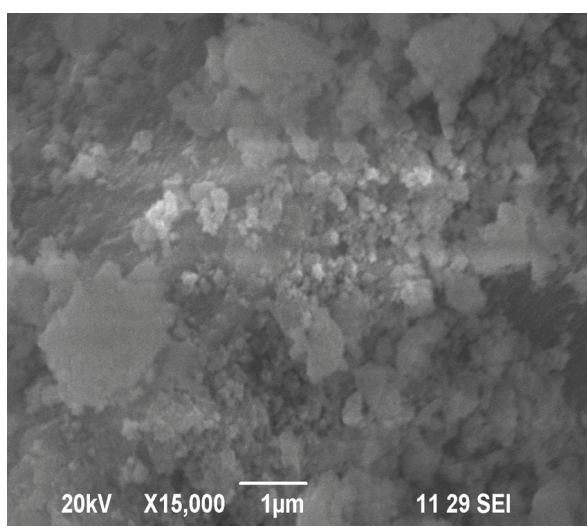

(f)

FIGURE 2: [(a)-(b)]: SEM images of coarse zirconia; [(c)-(f)]: SEM images of synthesized zirconium dioxide.

2.2. Characterization. The particle size distribution was analyzed by Fourier Transform Infrared Spectroscopy done using Schimadzu IR Prestige-21 using KBR pelletizer in the wavelength range of $400-4000 \mathrm{~cm}^{-1}$ with a resolution of $2 \mathrm{~cm}^{-1}$. X-ray diffraction was taken with Schimadzu, Japan Model XRD 6000 at room temperature using target of Cuk $\alpha$ with a voltage of $40.0(\mathrm{kV})$ and a current of $30.0(\mathrm{~mA})$ for $2 \theta$ between $10^{\circ}$ and $90^{\circ}$ with a sampling pitch of $1^{\circ}$ in scan mode of continuous scan and scan speed of 10 (deg/min). SEM microstructural analysis was performed using a JEOL JSM6390 microscope. The samples were ultrasonically dispersed in ethanol, deposited on a carbon film supported on a copper grid, and subjected for SEM analysis. TEM microstructural analysis was performed using JOEL JM 2010. Advanced analytical high resolution enables us to give morphological, chemical, and structure information. Electron diffraction 


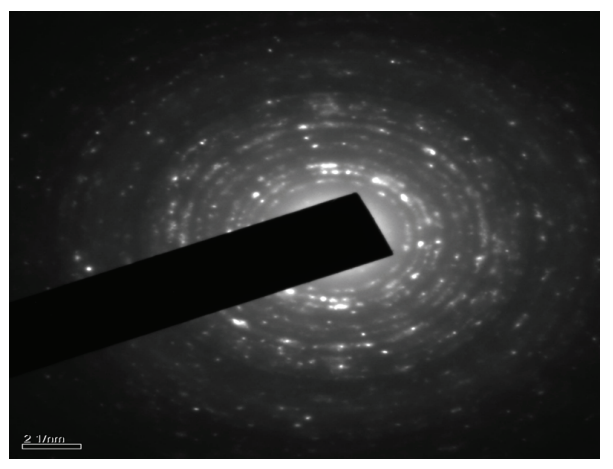

(a)

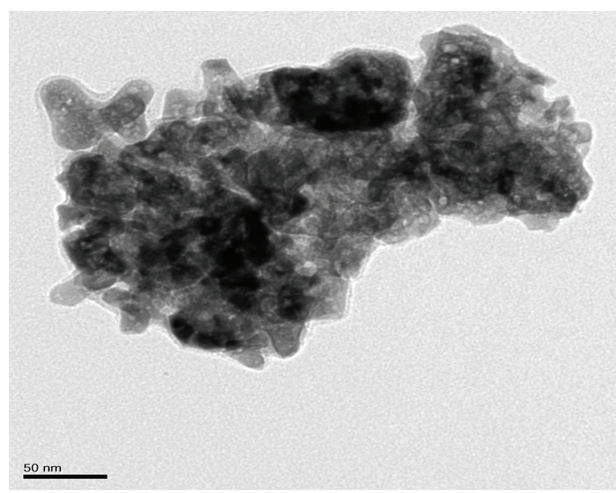

(c)

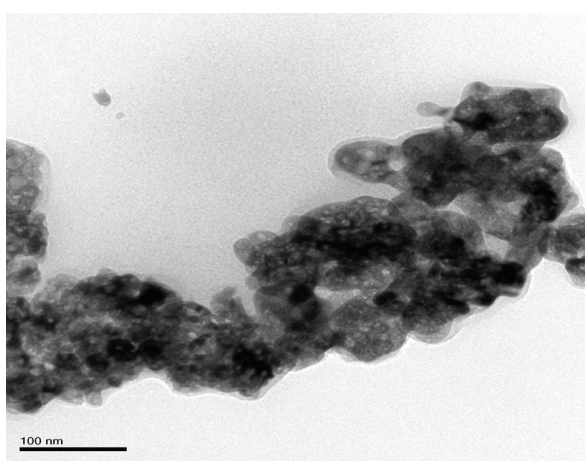

(b)

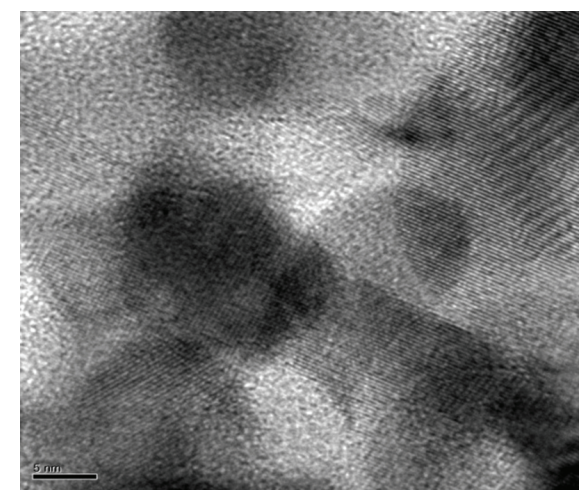

(d)

Figure 3: (a) Corresponding selected area diffraction, (b) $100 \mathrm{~nm}$, (c) $50 \mathrm{~nm}$, and (d) $5 \mathrm{~nm}$. TEM images of sol-gel synthesized zirconium dioxide.

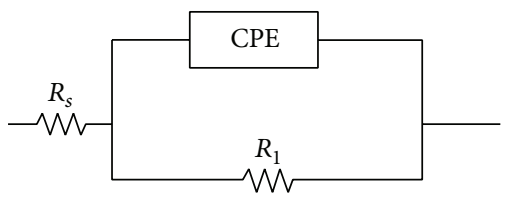

FIGURE 4: Equivalent circuit of impedance fit.

crystallographic studies were done on synthesized zirconia powder and SAED (selected area electron diffraction) pattern and CBED (convergent beam electron diffraction) pattern were taken for the sample. The sample was mixed with ethanol and ultrasonically vibrated for 5 minutes and it is coated on carbon coated copper grid and the powder is allowed to dry for 5 minutes before taking the patterns. For the impedance analysis, the powder is sprayed in a die of diameter $10 \mathrm{~mm}$ and the thickness of $1.29 \mathrm{~mm}$ applying a pressure of $200 \mathrm{Psi}$. The pellet was sintered at $300^{\circ} \mathrm{C}$ for $1 \mathrm{hr}$. Then, the pellet is calcined at various temperatures upto a maximum of $750^{\circ} \mathrm{C}$. Increase in processing temperatures (sintering and calcining) results in increase of grain size and reduction of pores or vacancies. Increase in temperature increases the grain sizewhich in turn influences the dielectric

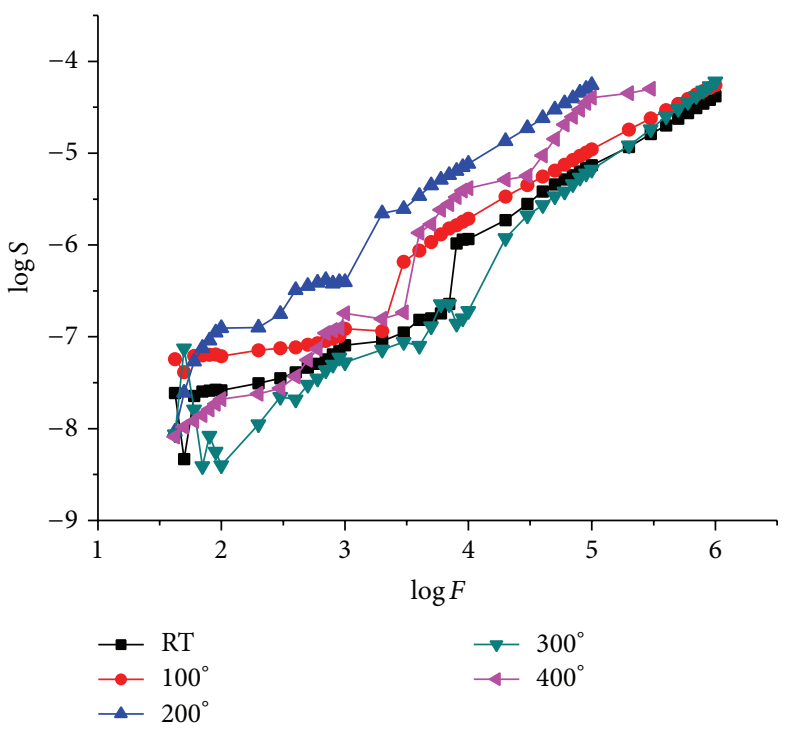

Figure 5: Comparison of conductivity graph versus $\log \sigma$ for the results ranging from room temperature to $400^{\circ} \mathrm{C}$.

and electrical properties of the sample. The growth of grain size is not seen after $750^{\circ} \mathrm{C}$ and, hence, the dielectric constant 


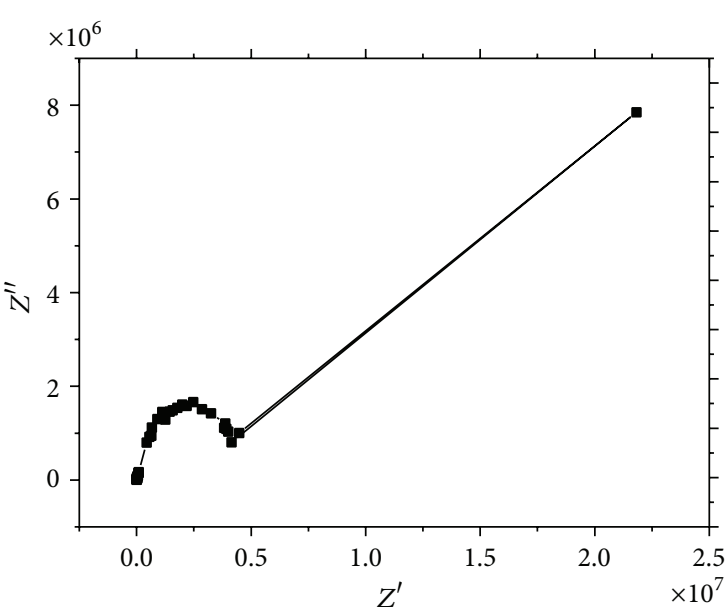

$-\square-\mathrm{RT}$

(a)

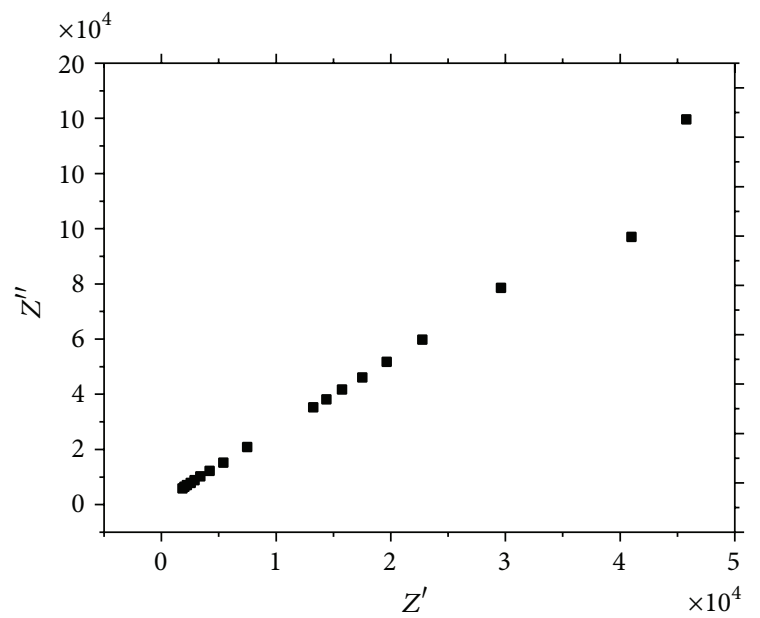

- $200^{\circ} \mathrm{C}$

(c)

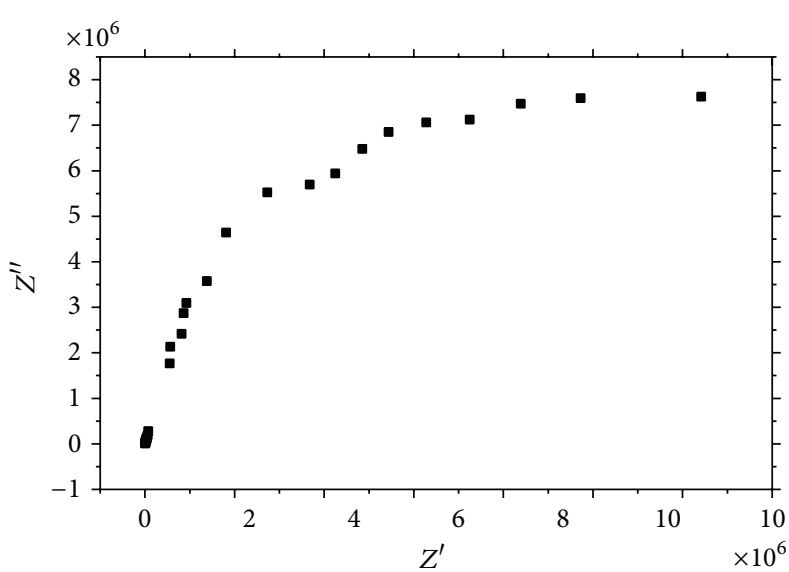

- $400^{\circ}$

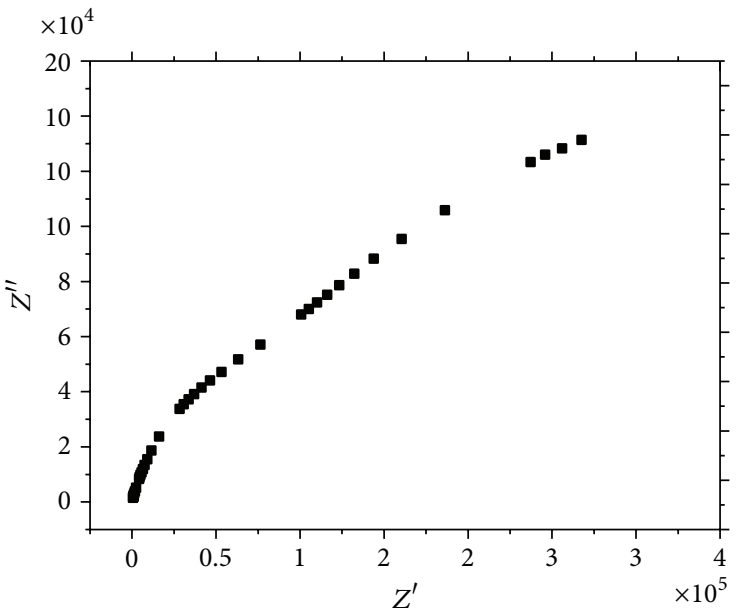

- $100^{\circ}$

(b)

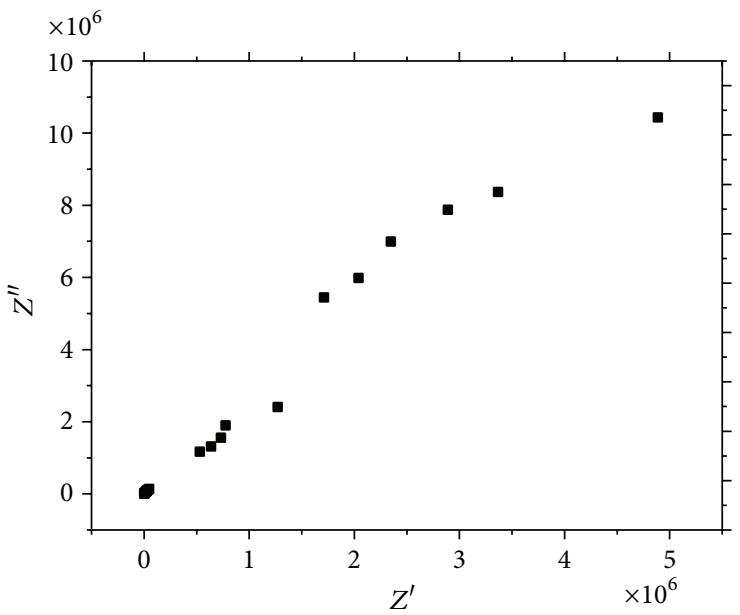

- $300^{\circ} \mathrm{C}$

(d)

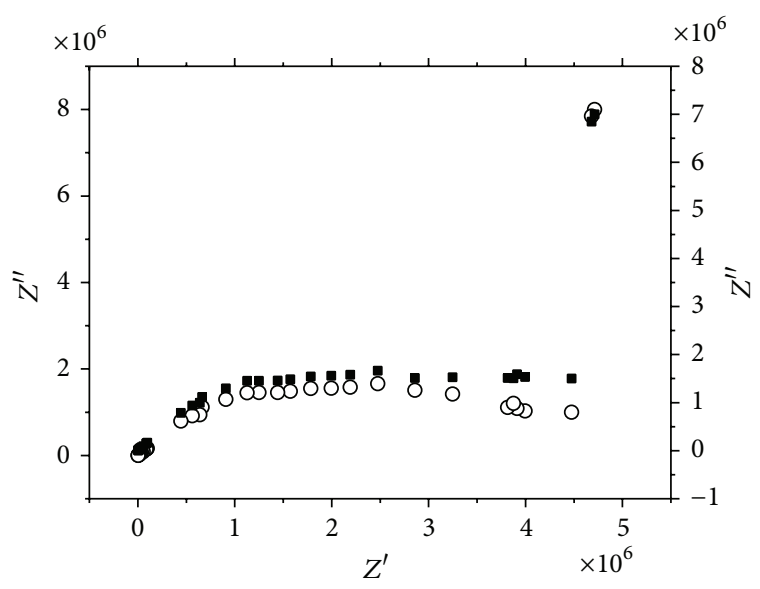

O Fit results for $450^{\circ} \mathrm{C}$

- Data for $450^{\circ} \mathrm{C}$

(e)

(f)

Figure 6: (a)-(e) Cole-cole plot of synthesized zirconia at different temperatures. (f) Impedance spectra plot of zirconium oxide with fit results at $450^{\circ} \mathrm{C}$. 
also remains constant after this temperature. The grain size growth was observed upto $750^{\circ} \mathrm{C}$ [19-23] and also it is confirmed that the grain did not grow bigger in size after $750^{\circ} \mathrm{C}$ [2]. This helps zirconia to have a constant dielectric. The impedance measurements were made with the Impedance Analyzer Hioki 3532-50 LCR Hi Tester with a frequency range of $42 \mathrm{~Hz}-5 \mathrm{MHz}$ at room temperature as well at elevated temperatures from $50^{\circ} \mathrm{C}$ to $550^{\circ} \mathrm{C}$ at the intervals of $50^{\circ} \mathrm{C}$. To avoid any diffusion problems, electrodes were not applied on the substrate but the sample was ensured to make complete contact with the electrodes by pressure contact.

\section{Results and Discussion}

The results of Fourier Transform Infrared Spectroscopy of coarse zirconia showed a strong peak of absorption at $1082.07 \mathrm{~cm}^{-1}$ and two weak peaks at $856.39 \mathrm{~cm}^{-1}$ and $877.61 \mathrm{~cm}^{-1}$ ensuring that the commercial sample is cubic zirconia; however, the synthesized zirconia after calcination at $750^{\circ} \mathrm{C}$ for two hours twice showed none of the major peaks except a small peak at $860.1 \mathrm{~cm}^{-1}$. Structural and morphological elucidations were checked using XRD (Figure 1), SEM (Figure 2), and TEM (Figure 3). The crystallite size of $\mathrm{ZrO}_{2}$ was estimated by XRD peaks. The reduction of size by sol-gel procedure was confirmed by different trials and all samples exhibit only peaks corresponding to typical $\mathrm{ZrO}_{2}$, [111], [200], and [220] planes. Using the diffraction peaks the crystallite size was calculated through Scherrer's equation $D=(0.9 \lambda / \beta$ $\cos \theta)$ and was found to fall in a range from $2 \mathrm{~nm}$ to $10 \mathrm{~nm}$.

The size of the particles of sol-gel synthesized zirconia, an average diameter of 50 to $80 \mathrm{~nm}$ particles sampled at random, was assessed from the corresponding SEM micrographs, whereas for coarse zirconia, the particle size was found to be in the range of $900 \mathrm{~nm}$ to $2.13 \mu \mathrm{m}$ (Figures 2(a) and 2(b)).

Coarse zirconia has a cubical structure which is inferred from the lattice parameters as indicated from JCPDS file " $\mathrm{a}=$ $\mathrm{b}=\mathrm{c}=5.129 \AA$ " with a crystallite size of $5.3 \mathrm{~nm}$ to $20 \mathrm{~nm}$ and particle size of $900 \mathrm{~nm}-2.13 \mu \mathrm{m}$ and the synthesized zirconia after calcination at $750^{\circ} \mathrm{C}$ for 4 hours showed monoclinic structure with the lattice parameters indicated from JCPDS file as “ $a=5.25, b=5.204$ and $c=5.213 \AA$ ” with particle size of $50 \mathrm{~nm}-80 \mathrm{~nm}$ and crystallite size of 2-10 $\mathrm{nm}$ determined by Debye-Scherrer method calculated using formula $D=k \lambda / \beta \cos \theta$. The XRD pattern (Figure 1) of resulting synthesized zirconia is of monoclinic structure that is stable up to $1100^{\circ} \mathrm{C}$ preferable for CMOS fabrication and it can replace $\mathrm{SiO}_{2}$ which has same stability. The SEM and TEM micrographs indicate that the $\mathrm{ZrO}_{2}$ nanoparticles after calcination were small, monoclinic crystallites in [111], [200], and [220] planes, and that they agglomerated to some extent because of nanocrystalline size and particles getting closer to each other. The obtained impedance spectra were fit to the theoretical equivalent circuit (Figure 4) using ZPlot software $[2,24]$. The values obtained from the fit and the actual data at $550^{\circ} \mathrm{C}$ are as follows. The equivalent circuit is obtained using the fit and consists of $R_{s}=1.5 \mathrm{~K} \Omega, R_{1}=3.05$ $\times 10^{6} \Omega, C_{\text {PEIT }}=1.174 \times 10^{-11} \mathrm{~F}$, and $C_{\text {PEIP }}=0.98$ with the peak frequency $f=3 \times 10^{4} \mathrm{~Hz}$, where $\omega \tau=1$. The capacitance and dielectric loss were also measured at this frequency and is found to be $C_{P}=1.7376 \times 10^{-11} \mathrm{~F}$. The same analysis has been made for all the temperatures from room temperature to $550^{\circ} \mathrm{C}$. The dielectric constant of zirconia was found from the formula $C=\epsilon_{0} \epsilon_{r} A / d$, where $\epsilon_{0}$ is permittivity of vacuum, $\epsilon_{r}$ is permittivity of zirconia, $A$ is Area of the pellet, and $d$ is diameter of the pellet. The calculated value of nanosized zirconium oxide obtained from the results is 25 . Also the conductivity values were verified using $\log \mathrm{F}$ versus $\log \sigma$ plot (Figure 5) and cole-cole plot (Figures 6(a)-2(f)) and the conductivity was found to be equal at room temperature and at $450^{\circ} \mathrm{C}$. The resistance was in the range of $10^{6}-10^{7}$ proving that the nanosized synthesized zirconia has good dielectric constant of 25 [2, 25-28].

\section{Conclusion}

In this paper, nanocrystallite monoclinic zirconia powder with particle size of $50-100 \mathrm{~nm}$ and crystallite size of $2 \mathrm{~nm}-$ $10 \mathrm{~nm}$ is obtained by sol-gel method, whereas coarse zirconia has a particle size of $1 \mu \mathrm{m}$ to $2 \mu \mathrm{m}$ with a crystallite size of $50 \mathrm{~nm}-90 \mathrm{~nm}$. The significant divergence of the phase composition, morphology, and the particle size has been found. The monoclinic phase is found to be predominant for the calcination temperatures up to $750^{\circ} \mathrm{C}$ [29]. The impedance profile of the nanosized zirconia gave a dielectric constant of 25 [2, 23, 26-28]. This work will be useful in the fabrication of nanosized zirconia as thin films to the required equivalent oxide thickness and analyzing the same for the high- $\kappa$ gate dielectric materials. When compared with $\mathrm{HfO}_{2}$, a rare earth metal, $\mathrm{ZrO}_{2}$, is cheaper and exhibits the same electrical properties as that of $\mathrm{HfO}_{2}$, which made it viable to be used in designing a high- $\kappa /$ metal gate, multigate MOSFET, with improved electrical characteristics.

\section{Conflict of Interests}

The authors declare that there is no conflict of interests regarding the publication of this paper.

\section{Acknowledgments}

The authors would like to thank Dr. Haris for the valuable suggestions and Karunya University, Coimbatore, for providing the facilities to carry out this work.

\section{References}

[1] M. R. H. Siddiqui, A. I. Al-Wassil, A. M. Al-Otaibi, and R. M. Mahfouz, "Effects of precursor on the morphology and size of $\mathrm{ZrO}_{2}$ nanoparticles, synthesized by sol-gel method in nonaqueous medium," Materials Research, vol. 15, no. 6, pp. 986989, 2012.

[2] D. Nirmal, B. Nalini, and P. Vijaya Kumar, "Nanosized high $\kappa$ dielectric material for FINFET," Integrated Ferroelectrics, vol. 121, no. 1, pp. 31-35, 2010.

[3] M. N. Tahir, L. Gorgishvili, J. Li et al., "Facile synthesis and characterization of monocrystalline cubic $\mathrm{ZrO}_{2}$ nanoparticles," Solid State Sciences, vol. 9, no. 12, pp. 1105-1109, 2007. 
[4] R. M. C. de Almeida and I. J. R. Baumvol, "Diffusion-reaction Modeling of silicon oxide interlayer growth during thermal annealing of high dielectric constant materials on silicon," Surface Science Reports, vol. 49, p. 1, 2003.

[5] J. A. Wang, M. A. Valenzuela, J. Salmones, A. Vázquez, A. García-Ruiz, and X. Bokhimi, "Comparative study of nanocrystalline zirconia prepared by precipitation and sol-gel methods," Catalysis Today, vol. 68, no. 1-3, pp. 21-30, 2001.

[6] M. Balog, M. Schieber, M. Michman, and S. Patai, "The chemical vapour deposition and characterization of $\mathrm{ZrO}_{2}$ films from organometallic compounds," Thin Solid Films, vol. 47, no. 2, pp. 109-120, 1977.

[7] B. Králik, E. K. Chang, and S. G. Louie, "Structural properties and quasiparticle band structure of zirconia," Physical Review B, vol. 57, no. 12, pp. 7027-7036, 1998.

[8] P. Abelard and J. Baumard, "The electrical conductivity of cubic stabilized zirconia," Pure and Applied Chemistry, vol. 67, no. 11, pp. 1891-1940, 1995.

[9] M. S. Lundstrom and J. Guo, Nano Scale Transistors: Device Physics, Modelling and Simulation, vol. 39 of Silicon Moore's Law, Springer, New York, NY, USA, 2006.

[10] P. M. Zeitzoff, J. A. Hutchby, G. Bersuker, and H. R. Huff, "Integrated circuit technologies: from conventional CMOS to nanoscale era," in Nano and Giga Challenges in Microelectronics, J. Greer, A. Korkin, and J. Labanowski, Eds., pp. 1-25, Elsevier, New York, NY, USA, 2003.

[11] http://www.itrs.net/Links/2008ITRS/Home2008.htm.

[12] C. Jeffrey Brinker and G. W. Scherer, Sol-Gel Science: The Physics and Chemistry of Sol-Gel Processing, 1990.

[13] L. E. Davies, N. A. Bonini, S. Locatelli, and E. E. Gonzo, "Characterization and catalytic activity of zirconium dioxide prepared by sol-gel," Latin American Applied Research, vol. 35, no. 1, pp. 23-28, 2005.

[14] M. D. Curran, D. D. Pooré, and A. E. Stiegman, "Vanadiasilica sol-gel derived glass: factors affecting homogeneity and morphology," Chemistry of Materials, vol. 10, no. 10, pp. 31563166, 1998.

[15] K. Yanagisawa, Y. Yamamoto, Q. Feng, and N. Yamasaki, "Formation mechanism of fine anatase crystals from amorphous titania under hydrothermal conditions," Journal of Materials Research, vol. 13, no. 4, pp. 825-829, 1998.

[16] Z. Zhan and H. C. Zeng, "A catalyst-free approach for sol-gel synthesis of highly mixed $\mathrm{ZrO}_{2}-\mathrm{SiO}_{2}$ oxides," Journal of NonCrystalline Solids, vol. 243, no. 1, pp. 26-38, 1999.

[17] V. Santos, M. Zeni, C. P. Bergmann, and J. M. Hohemberger, "Correlation between thermal treatment and tetrago$\mathrm{nal} /$ monoclinic nanostructured zirconia powder obtained by sol-gel process," Reviews on Advanced Materials Science, vol. 17, no. 1-2, pp. 62-70, 2008

[18] C. J. Brinker, G. C. Frye, A. J. Hurd, and C. S. Ashley, "Fundamentals of sol-gel dip coating," Thin Solid Films, vol. 201, no. 1, pp. 97-108, 1991.

[19] G. D. Wilk, R. M. Wallace, and J. M. Anthony, "High- $\kappa$ gate dielectrics: current status and materials properties considerations," Journal of Applied Physics, vol. 89, no. 10, pp. 5243-5275, 2001.

[20] A. Manan, Y. Iqbal, and I. Qazi, "The effect of sintering temperature on phase, microstructure and properties of $\mathrm{Sr}_{5} \mathrm{Nb}_{4} \mathrm{TiO}_{17}$, Journal of Physics, vol. 241, no. 1, Article ID 012028, 2010.

[21] R. Pandu, K. L. Yadav, A. Kumar, P. Ravinder Reddy, and A. V. S. S. K. S. Gupta, "Effect of sintering temperature on structural and electrical properties of $\mathrm{BiFeO}_{3}$ multiferroics," Indian Journal of Engineering and Materials Sciences, vol. 17, no. 6, pp. 481-485, 2010.

[22] G.-Y. Guo and Y.-L. Chen, "A nearly pure monoclinic nanocrystalline zirconia," Journal of Solid State Chemistry, vol. 178, no. 5, pp. 1675-1682, 2005.

[23] K. Geethalakshmi, T. Prabhakaran, and J. Hemalatha, "Dielectric studies on nano zirconium dioxide synthesized through coprecipitation process, world academy of science," Engineering and Technology, vol. 64, pp. 179-182, 2012.

[24] http://www.gamry.com/.

[25] D. Nirmal, P. Vijayakumar, D. M. Thomas, B. K. Jebalin, and N. Mohankumar, "Subthreshold performance of gate engineered FinFET devices and circuit with high K dielectrics," Microelectronics Reliability, vol. 53, pp. 499-504, 2013.

[26] P. J. Harrop and J. N. Wanklyn, "The dielectric constant of zirconia," British Journal of Applied Physics, vol. 18, no. 6, article 305, pp. 739-742, 1967.

[27] Y. Ma, Y. Ono, L. Stecker, D. R. Evans, and S. T. Hsu, "Zirconium oxide based gate dielectrics with equivalent oxide thickness of less than $1.0 \mathrm{~nm}$ and performance of submicron MOSFET using a nitride gate replacement process," in Proceedings of the IEEE International Devices Meeting (IEDM '99), pp. 149-152, December 1999.

[28] J. Robertson, "High dielectric constant oxides," The European Physical Journal, vol. 28, no. 3, pp. 265-291, 2004.

[29] S. Asadi, H. Abdizadeh, and Y. Vahidshad, "Effect of crystalline size on the structure of copper doped zirconia nanoparticles synthesized via sol-gel," Journal of Nanostructures, vol. 2, pp. 205-212, 2012. 

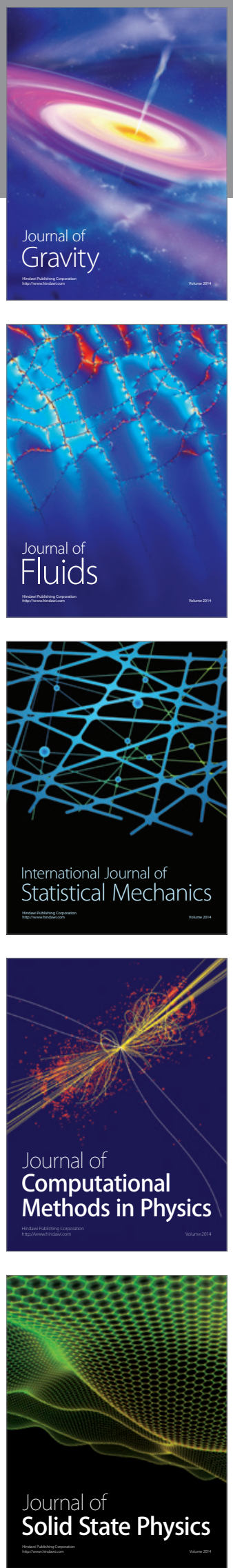

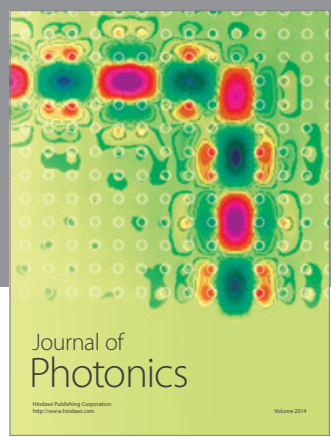

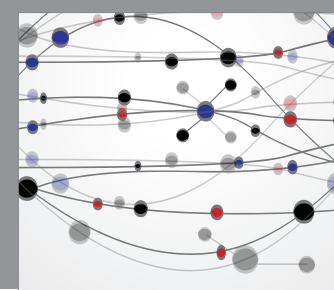

The Scientific World Journal

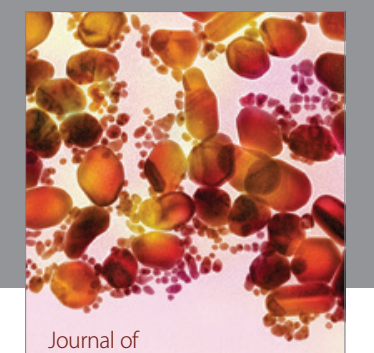

Soft Matter
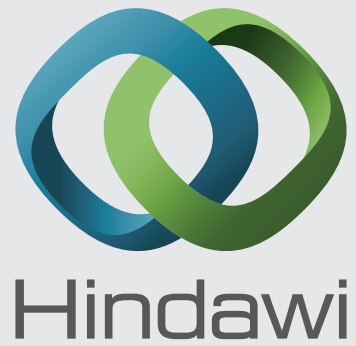

Submit your manuscripts at

http://www.hindawi.com
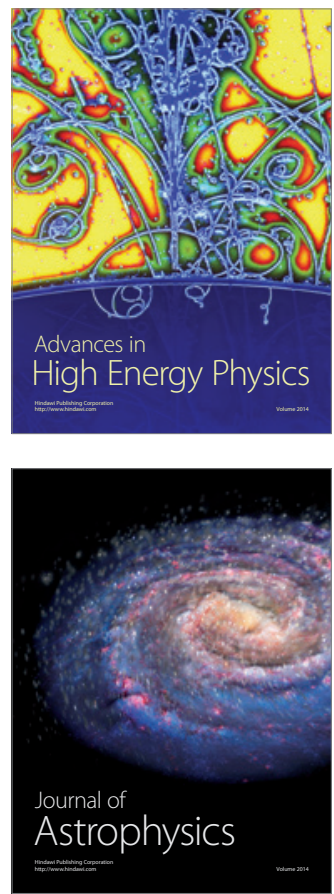
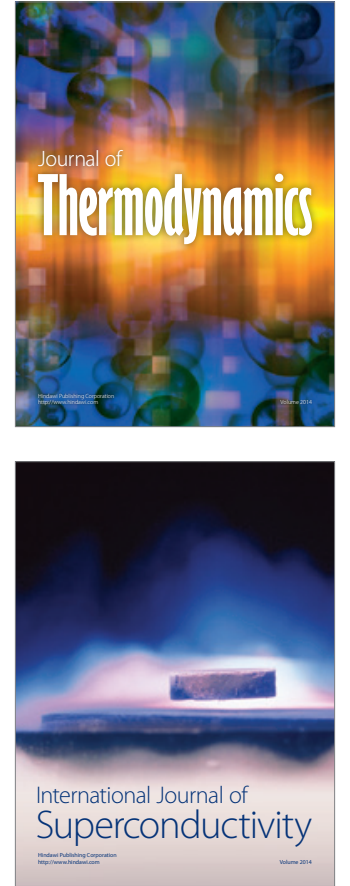
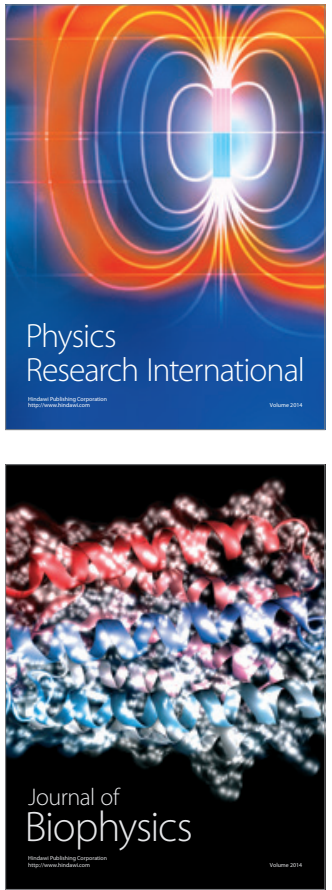
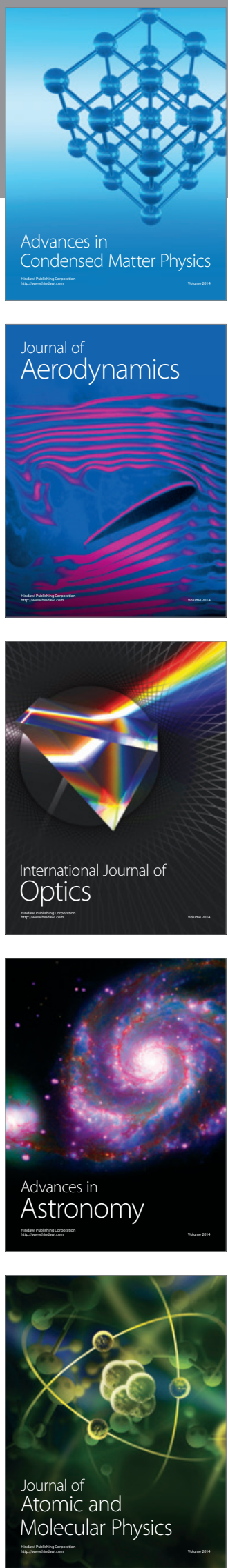